\title{
Tank Drainage for an Electrically Conducting Newtonian Fluid with the use of the Bessel Function
}

\author{
Mansoor Ali Khaskheli \\ Faculty of Bio-Sciences \\ SBBUVAS \\ Sakrand, Pakistan \\ mansoor11ms21@gmail.com
}

\author{
Kamran Nazir Memon \\ Department of Mathematics and Statistics \\ QUEST \\ Nawabshah, Pakistan \\ kamrannazirmemon@gmail.com
}

\author{
Abdul Hanan Sheikh \\ Department of Mathematics and Statistics \\ QUEST \\ Nawabshah, Pakistan \\ hanangul12@yahoo.com
}

\author{
Abdul Majeed Siddiqui \\ Pennsylvania State University \\ York Campus \\ USA \\ ams5@psu.edu
}

\author{
Syed Feroz Shah \\ Department of Basic Science \& Related Studies \\ MUET \\ Jamshoro, Pakistan \\ feroz.shah@faculty.muet.edu.pk
}

\begin{abstract}
In this work, an unsteady flow for drainage through a circular tank of an isothermal and incompressible Newtonian magnetohydrodynamic (MHD) fluid has been investigated. The series solution method is employed, and an analytical solution is obtained. Expressions for velocity field, average velocity, flow rate, depth of fluid at different times in the tank and time required for the wide-ranging drainage of the fluid (time of efflux) have been obtained. The Newtonian solution is attained by assuming $\sigma B_{0}{ }^{2}=0$. The effects of various developing parameters on velocity field $v_{z}$ and depth of fluid $H(t)$ are presented graphically. The time needed to drain the entire fluid and its depth are related and such relations are obtained in close form. The effect of electromagnetic forces is analyzed. The fluid in the tank will drain gradually and it will take supplementary time for complete drainage.
\end{abstract}

Keywords-tank drainage; Newtonian MHD fluid; analytical solution; series solution

\section{INTRODUCTION}

Tank seepage by gravity is an old, but still open issue. The tank might be exhausted through an opening (gap situation) or may be depleted through a connected pipe. The pipe may be flat or vertical or may include a full directing system with vertical drop and even enlargement with valve and fittings, etc. The tank has frequently a barrel shaped structure with a vertical divider. Anyway the base level may be cone molded, hemispherical or of another shape. Gravity depleting of liquid frameworks is utilized quite often in businesses, e.g. in condensate depleting, water division, recovery of synthetic concoctions from a homestead tank, etc. The outfitted model will precisely show the tank depleting conduct for all tanks with a similar arrangement. End impacts, exactness of time estimation, precision of stature estimations and grating misfortunes will be examined [1]. Many real life applications are modeled by the tank drainage flow. Considering the inevitable applications, this problem has attracted research of not only mathematical but also engineering background. Authors in [2] have used the Newtonian fluid principle for tank drainage flow and power law fluid has been considered in [3] in an attempt to solve the problem analytically. Theoretical results of time drainage have been derived in $[3,4]$. Further, in [5], the study has been extended while considering different geometries for outflow. In $[3,6]$ the discharge velocity and flow rate of the fluid outflowing through a hole are discussed using Torricelli's equation. The relationship between the efflux time needed to drain and the depth of the turbulent fluid has been derived in $[7,8]$. The unsteady tank drainage flows are discussed in $[9,10]$ in two and three dimensional tanks respectively. Efflux time and the cylindrical flow are presented in [11]. The gravitational force has been considered in [12], acting on a fluid in a spherical tank. In some similar work regarding gravity driven flow [13], the polymer solutions have been considered for drag reduction.

An exact solution of Navier Stokes equation modeling the tank drainage flow problem for an electrically conducting Newtonian fluid has been derived in [14]. The exact solution for a tank with a Newtonian fluid with slip condition has been solved in [16]. In [17], an analytical solution approach of the tank drainage problem for an electrically conducting power law fluid is presented. In [18-20], the Ellis fluid has been studied regarding the tank drainage problem with the special cases of Newtonian, power law and Bingham plastic fluid.

\section{BASIC EQUATIONS}

The governing equations for the incompressible thick liquid stream, dismissing warm impacts are:

$$
\begin{gathered}
\nabla \cdot \boldsymbol{V}=0 \\
\rho \frac{D \boldsymbol{V}}{D t}=-\nabla p+\rho \boldsymbol{b}+\nabla \cdot \boldsymbol{T}+(\boldsymbol{J} \times \boldsymbol{B})
\end{gathered}
$$


where $\boldsymbol{V}$ represents the velocity vector, $\rho$ represents density, $p$ is the dynamic pressure, $\boldsymbol{b}$ the body force and $\boldsymbol{T}$ the extra stress tensor. The material derivative is denoted by $\frac{D}{D t}$ and the electric current density is denoted by $\boldsymbol{J}$. Also $\boldsymbol{B}=\boldsymbol{B}_{\circ}+\boldsymbol{b}$ (where $\boldsymbol{B}, \boldsymbol{B}$ 。 and $\boldsymbol{b}$ are the total magnetic field, the forced magnetic field, and the induced magnetic field respectively). The modified Ohm's law and Maxwell's equation [21-24] are:

$$
\begin{gathered}
J=\sigma[E+V \times B] \\
\operatorname{div} B=0, \nabla \times B=\mu_{m} J, \operatorname{curl} E=-\frac{\partial B}{\partial t}
\end{gathered}
$$

where $\boldsymbol{E}, \boldsymbol{\mu}_{\boldsymbol{m}}$ and $\sigma$ are the electric field, the magnetic permeability, and the electrical conductivity respectively. The magnetic flux $\boldsymbol{B}$ is derived by the magnetic induction equation which describes the motion of an electrically conducting fluid under the influence of a magnetic field. The magnetic field $\boldsymbol{B}$ is assumed to be perpendicular to the velocity field $\boldsymbol{V}$. The magnetic Reynolds number is very small, which means that the induced magnetic field $\boldsymbol{b}$ is negligible in comparison with $\boldsymbol{B}$. Meanwhile no outdoor electric field is considered, the outcome of polarization of the ionized fluid is insignificant, and the fluid flow area is supposed to be free of electric field. Under these expectations [25], the MHD force in (2) can be placed into:

$$
J \times B=-\sigma B_{\circ}^{2} \boldsymbol{V}
$$

The extra stress tensor, essential in a Newtonain fluid is specified by:

$$
\boldsymbol{T}=\mu \boldsymbol{A}_{1}
$$

where $\boldsymbol{A}_{1}$ is the first Rivlin-Ericksen tensor defined as:

$$
\boldsymbol{A}_{1}=\nabla \boldsymbol{V}+(\nabla \boldsymbol{V})^{T}
$$

For the Rivilin-Ericksen tensor, in cylindrical co-ordinates, we have:

$$
\nabla \mathrm{V}=\left[\begin{array}{ccc}
\frac{\partial u}{\partial r} & \frac{1}{r}\left[\frac{\partial u}{\partial \theta}-v\right] & \frac{\partial u}{\partial z} \\
\frac{\partial v}{\partial r} & \frac{1}{r}\left[\frac{\partial v}{\partial \theta}+u\right] & \frac{\partial v}{\partial z} \\
\frac{\partial w}{\partial r} & \frac{1}{r} \frac{\partial w}{\partial \theta} & \frac{\partial w}{\partial z}
\end{array}\right]
$$

\section{TANK DRAINAGE}

Consider a cylinder-shaped tank containing an incompressible Newtonian MHD fluid. The radius of the tank is $R_{T}$ and its diameter is $D$. The initial depth of the fluid is $H_{0}$. The fluid in the tank is drained down through a pipe of radius $R$ and length $L$. Let $H(t)$ be the depth of fluid in the tank at any time $t$. We plan to compute flow rate, velocity profile, shear stress, pressure profile, average velocity on the pipe, connection of how does the time vary with length, and the time required for the whole drainage. We use cylindrical coordinates $(r, \theta, z)$ with $z$-axis end to end the midpoint of the pipe in vertical direction and $r$-axis normal to it. Then the flow is only in the $z$-direction, and the $r$ and $\theta$ components of velocity field are zero:

$$
V=\left[v_{r}, v_{\theta}, v_{z}\right]=\left[0,0, v_{z}(r, t)\right]
$$

By (9), the equation of continuity (1) is identically fulfilled and the momentum equation (2) after using (3), reduces toward:

r-component of momentum:

$$
\frac{\partial p}{\partial r}=0
$$

$\theta$-component of momentum:

$$
\frac{1}{r} \frac{\partial p}{\partial \theta}=0
$$

Z-component of momentum:

$$
\rho \frac{\partial V_{Z}}{\partial t}=-\frac{\partial p}{\partial z}+\frac{\eta}{r} \frac{\partial}{\partial r}\left(r \frac{\partial V_{Z}}{\partial r}\right)+\rho g-\sigma B_{0}^{2} V_{Z}(r)
$$

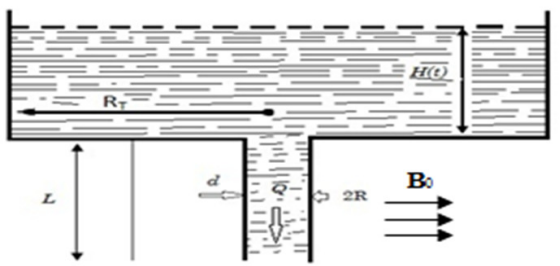

Fig. 1. Geometry of the tank drainage flow through the pipe

From (10)-(12) we can see that the equation of motion is simple, yielding that the pressure is only a function of $z$ and $t$ and the equation to be solved for $v z(r, t)$ is:

$$
\rho \frac{\partial V_{z}}{\partial t}=-\frac{\partial p}{\partial z}+\frac{\eta}{r} \frac{\partial}{\partial r}\left(r \frac{\partial V_{z}}{\partial r}\right)+\rho g-\sigma B_{0}^{2} V_{z}(r)
$$

Equation (13) is a partial differential equation for $p$ and $v_{z}$. The velocity in the pipe flow remains almost constant in time due to the slow draining since we may neglect the time derivative $\frac{\partial v_{z}}{\partial t}$. The flow in the pipe of radius $R$ is caused by both gravity and hydrostatic pressure. The pressures at the pipe entry and exit are one-to-one: at $z=0, p=p_{1}=\rho g H(t)$, at $z=L, P=P_{2}=0$, so that:

$$
\frac{\partial p}{\partial z}=-\frac{\rho g H(t)}{L}
$$

The domain of the problem is bounded by the following equation (boundary conditions):

$$
\begin{aligned}
\frac{1}{r} \frac{\partial}{\partial r}\left(r \frac{\partial v_{Z}}{\partial r}\right)-\frac{\sigma B_{0}{ }^{2}}{\eta} V_{Z}(r) & =-\frac{\rho g}{\eta}\left[\frac{H(t)}{L}+1\right] \\
\text { at } r & =0, \frac{\partial v_{Z}}{\partial r}=0 \\
\text { at } r & =R, V_{Z}=0
\end{aligned}
$$

Equation (15) can be written as:

$$
\frac{\partial^{2} v_{Z}}{\partial r^{2}}+\frac{1}{r} \frac{\partial v_{Z}}{\partial r}-\frac{\sigma B^{2}{ }_{0}}{\eta} V_{Z}(r)=-\frac{\rho g}{\eta}\left[\frac{H(t)}{L}+1\right]
$$

with:

$$
v_{z}=\frac{\rho g}{\sigma B^{2}{ }_{0}}\left[\frac{H(t)}{L}+1\right]-G(r)
$$

By using (19) in (16)-(18), we obtain:

$$
\frac{\partial^{2} G(r)}{\partial r^{2}}+\frac{1}{r} \frac{\partial G(r)}{\partial r}-\frac{\sigma B^{2} 0}{\eta} G(r)=0
$$


With related boundary conditions:

$$
\begin{gathered}
\text { at } r=0, \frac{\partial G(r)}{\partial r}=0 \\
\text { at } r=R, G(r)=\frac{\rho g}{\sigma B^{2}{ }_{0}}\left[\frac{H(t)}{L}+1\right]
\end{gathered}
$$
is:

The general solution of (20) after using the Bessel function

$$
G(r)=C_{1} J_{0}\left(r B_{0} \sqrt{\frac{-\sigma}{\eta}}\right)+C_{2} Y_{0}\left(-r B_{0} \sqrt{\frac{-\sigma}{\eta}}\right)
$$

where $C_{1}$ and $C_{2}$ are arbitrary constants and $J_{0}$ and $Y_{0}$ are the Bessel functions of first and second kind of order zero. For a particular solution using (21)-(22) in (23), we get:

$$
G(r)=\frac{\rho g(H(t)+L) J_{0}\left(r B_{0} \sqrt{\frac{-\sigma}{\eta}}\right)}{L \sigma J_{0}\left(R B_{0} \sqrt{\frac{-\sigma}{\eta}}\right) B^{2}{ }_{0}}
$$

Substituting (24) in (19) we get:

$$
v_{z}=\frac{\rho g(H(t)+L)}{\sigma J_{0}\left(R B_{0} \sqrt{\frac{-\sigma}{\eta}}\right) B^{2}{ }_{0}}\left(J_{0}\left(R B_{0} \sqrt{\frac{-\sigma}{\eta}}\right)-J_{0}\left(r B_{0} \sqrt{\frac{-\sigma}{\eta}}\right)\right)
$$

\section{A. Finding Flow Rate (Q)}

According to (26) we can find the flow rate:

$$
\mathcal{Q}=\int_{0}^{\mathrm{R}} 2 \pi r \mathrm{v}_{\mathrm{z}}(\mathrm{r}, \mathrm{t}) \mathrm{dr}
$$

By using the velocity profile from (25) in (26), we get:

$$
\mathcal{Q}=\frac{\rho g \pi R^{4}(H(t)+L) 0 \tilde{F}_{1}\left(; 3 ; \frac{R^{2} \sigma B^{2} 0}{4 \eta}\right)}{4 \eta L I_{0}\left(R B_{0} \sqrt{\frac{\sigma}{\eta}}\right)}
$$

where $I_{0}$ is the modified Bessel function of the $1^{\text {st }}$ kind of order 0 and here $0 \tilde{\mathrm{F}}_{1}\left(; 3 ; \frac{R^{2} \gamma^{2}}{4}\right)$ presents the Hyper geometric $0 \tilde{F}_{1}$ regularized function, which can be specified as:

$$
0 \tilde{F}_{1}\left(; 3 ; \frac{R^{2} \sigma B^{2}{ }_{0}}{4 \eta}\right)=\sum_{k=0}^{\infty}\left(\frac{R^{2} \sigma B^{2}{ }_{0}}{4 \eta}\right)^{\mathrm{k}} \frac{1}{\Gamma(3+k) k !}
$$

\section{B. Finding Average Velocity}

We find the average velocity $\bar{V}$ by:

$$
\bar{V}=\frac{Q}{\pi R^{2}}
$$

Putting (27) in (29) we get:

$$
\bar{V}=\frac{\rho g \mathrm{R}^{2}(\mathrm{H}(\mathrm{t})+\mathrm{L}) 0 \widetilde{\mathrm{F}}_{1}\left(; 3 ; \frac{\mathrm{R}^{2} \sigma \mathrm{B}^{2} 0}{4 \eta}\right)}{4 \eta \mathrm{LI}_{0}\left(\mathrm{RB}_{0} \sqrt{\frac{\sigma}{\eta}}\right)}
$$

\section{Finding Mass Balance}

We find the mass balance by:

$$
\frac{d}{d t}\left[\pi R^{2}{ }_{T} H(t)\right]=-\mathcal{Q}(\mathrm{t})
$$

The remaining mass in the tank (mass balance) can be computed by the following procedure:

Putting (27) in (31), and then separating variables on both sides of equation one obtains by taking $\mathrm{t}=0, H(t)=H_{0}$ :

$$
H(t)=-L+e^{\frac{-\rho g R^{4} t_{0} \tilde{F}_{1}\left(; 3 ; \frac{R^{2} \sigma B_{0}^{2}}{4 \eta}\right)}{4 \eta L R_{T}^{2} I_{0}\left(R B_{0} \sqrt{\frac{\sigma}{n}}\right)}}\left(H_{0}+L\right)
$$

The relation of tank depth with respect to time is:

$$
\frac{-4 \eta L R^{2} T I_{0}\left(R B_{0} \sqrt{\frac{\sigma}{\eta}}\right)}{\rho g R^{4} 0 \widetilde{\mathrm{F}}_{1}\left(; 3 ; \frac{\mathrm{R}^{2} \sigma \mathrm{B}^{2} 0}{4 \eta}\right)} \ln \left(\frac{H(t)+L}{H_{0}+L}\right)=t
$$

The time of efflux is attained by taking $H(t)=0$ in (33):

$$
\frac{4 \eta L R^{2} T I_{0}\left(R B_{0} \sqrt{\frac{\sigma}{\eta}}\right)}{\rho g R^{4} 0 \widetilde{\mathrm{F}}_{1}\left(; 3 ; \frac{\mathrm{R}^{2} \sigma \mathrm{B}^{2} 0}{4 \eta}\right)} \ln \left(\frac{H_{0}}{L}+1\right)=t
$$

Remark: By taking $\sigma B^{2}{ }_{0}=0$ in (34), the Newtonian arrangement without MHD is recovered, which remains complete through by Bernoulli's equation [26].

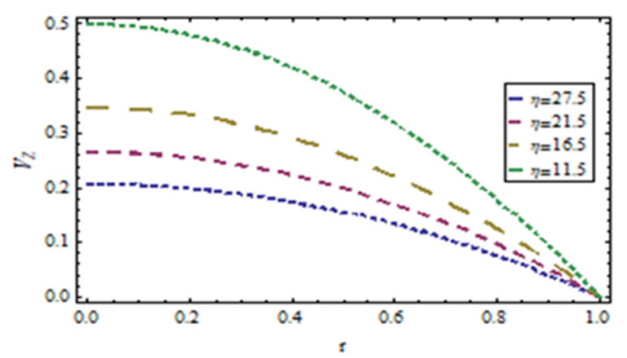

Fig. 2. Effect of $\eta$ on velocity profile , when $\rho=0.78 \mathrm{~g} / \mathrm{cm}^{3}, R=1 \mathrm{~cm}$, $g=9.8 \mathrm{~m} / \mathrm{s}^{2}, L=10 \mathrm{~cm}, H(t)=20 \mathrm{~cm}, \sigma=0.1, B_{0}=0.25$

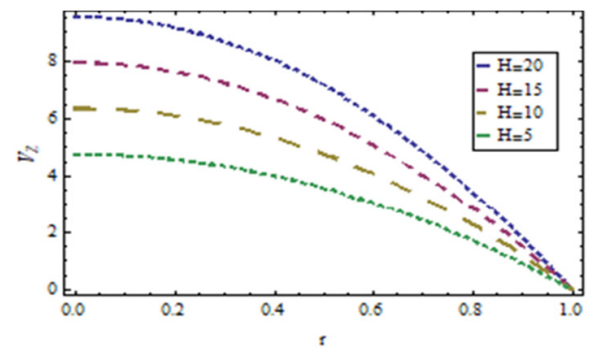

Fig. 3. Effect of $H(t)$ on velocity profile , when $\eta=0.6$ poise, $\rho=0.78 \mathrm{~g} / \mathrm{cm}^{3}$, $R=1 \mathrm{~cm}, g=9.8 \mathrm{~m} / \mathrm{s}^{2}, L=10 \mathrm{~cm}, \sigma=0.1, B_{0}=0.25$

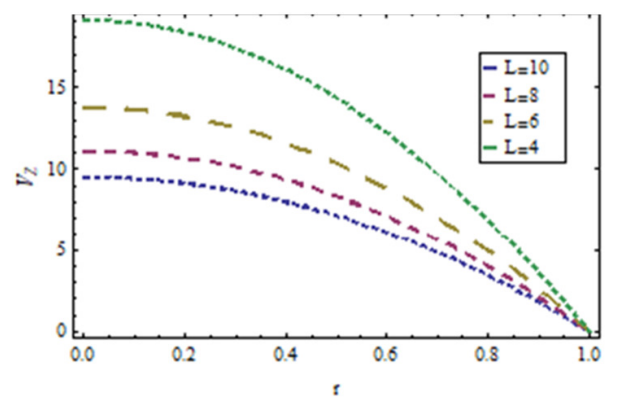

Fig. 4. Effect of $L$ on velocity profile, when $\eta=0.6$ poise, $\rho=0.78 \mathrm{~g} / \mathrm{cm}^{3}$, $R=1 \mathrm{~cm}, g=9.8 \mathrm{~m} / \mathrm{s}^{2}, H(t)=20 \mathrm{~cm}, \sigma=0.1, B_{0}=0.25$ 


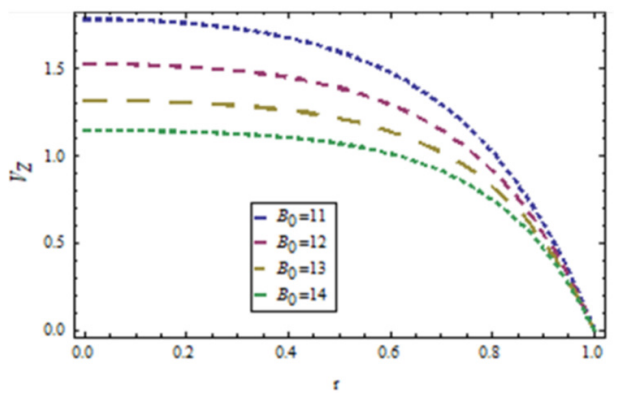

Fig. 5. Effect of $B_{0}$ on velocity profile, when $\eta=0.6$ poise, $\rho=0.78 \mathrm{~g} / \mathrm{cm}^{3}$, $R=1 \mathrm{~cm}, g=9.8 \mathrm{~m} / \mathrm{s}^{2}, L=10 \mathrm{~cm}, H(t)=20 \mathrm{~cm}, \sigma=0.1$

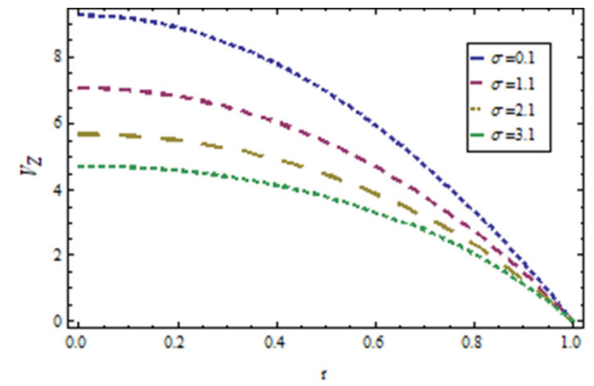

Fig. 6. Effect of $\sigma$ on velocity profile, when $\eta=0.6$ poise, $\rho=0.78 \mathrm{~g} / \mathrm{cm}^{3}$, $R=1 \mathrm{~cm}, g=9.8 \mathrm{~m} / \mathrm{s}^{2}, L=10 \mathrm{~cm}, H(t)=20 \mathrm{~cm}, B_{0}=1$

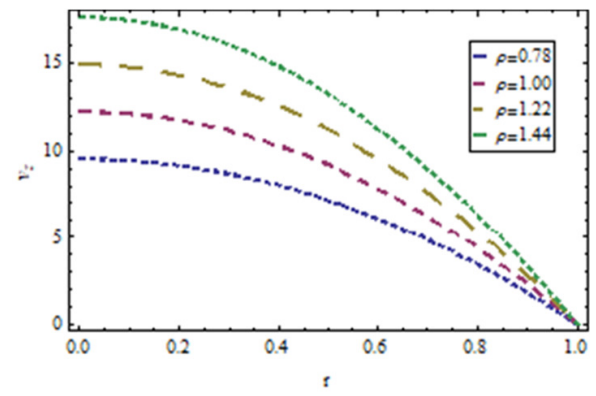

Fig. 7. Effect of $\rho$ on velocity profile, when $R=1 \mathrm{~cm}, \quad \eta=0.6$ poise, $g=9.8 \mathrm{~m} / \mathrm{s}^{2}, L=10 \mathrm{~cm}, \mathrm{H}(t)=20 \mathrm{~cm}, \sigma=0.1, B_{0}=0.25$

\section{RESULTS AND DISCUSSION}

In this article, the unsteady drainage problem through a circular pipe which is attached at the center of the circular tank by applying an incompressible, isothermal electrically conducting Newtonian fluid was addressed, through which analytical solutions of Bessel's differential equation were obtained, which are certainly more accurate than the solutions obtained with existing methods like ADM and perturbation methods. The analytical solution was obtained in a compact form, which helps in visualizing the fluid velocity profile, flow rate, average velocity, and time required for complete drainage. This can be utilized in many applications, for example waste management, industrial flow problems, etc. The effect of velocity profile $V_{Z}$ and depth $H(t)$ has been analyzed with regard to various parameters. On the velocity profile, the effects of different parameters were observed, i.e. the effect of the applied magnetic field $B_{0}$, electrical conductivity $\sigma$, pipe radius $R$, depth $H(t)$, density $\rho$, dynamic viscosity $\eta$, length of pipe $L$ and the difference of the radius of the circular tank $R_{T}$ on the depth of tank $H(t)$ versus the radius of the pipe $R$ and time $t$. It was observed that the magnitude of the velocity of the fluid decreases when the applied magnetic field $B_{0}$, length of the pipe $L$, electrical conductivity $\sigma$, dynamic viscosity $\eta$ increase (Figures 2-7) and increases when the depth $H(t)$ and density $\rho$ of the fluid increase Figures (8-9).

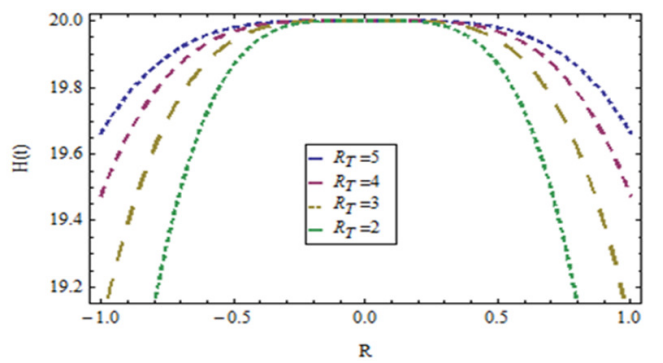

Fig. 8. Effect of $R_{T}$ on depth w.r.to $R$, when $\eta=0.6$ poise, $\rho=1.38 \mathrm{~g} / \mathrm{cm}^{3}$, $g=9.8 \mathrm{~m} / \mathrm{s}^{2}, L=10 \mathrm{~cm}, t=1, H_{0}=20 \mathrm{~cm}, \sigma=0.1, B_{0}=0.25$

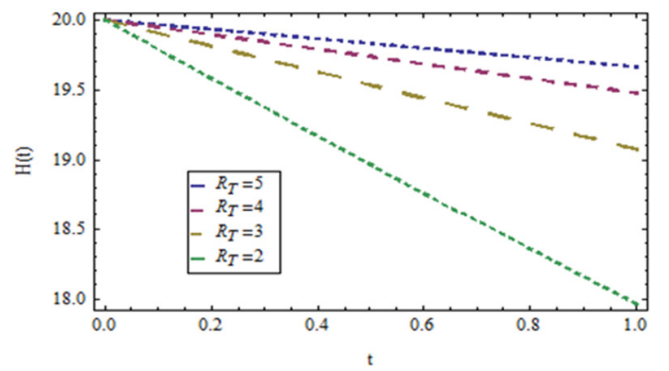

Fig. 9. Effect of $R_{T}$ on depth w.r.to $t$, when $\eta=0.6$ poise, $\rho=1.38 \mathrm{~g} / \mathrm{cm}^{3}$, $g=9.8 \mathrm{~m} / \mathrm{s}^{2}, L=10 \mathrm{~cm}, R=1 \mathrm{~cm}, H_{0}=20 \mathrm{~cm}, \sigma=0.1, B_{0}=0.25$

\section{CONCLUSION}

The equation of isothermal, incompressible, unsteady tank drainage flow for an electrically conducting Newtonian fluid is studied to obtain closed form expressions of velocity field, average velocity, flow rate, fluid depth in the tank, and time required for complete drainage of the fluid (time of efflux) by using Bessel functions. A relationship between the fluid depth and time required to complete drainage in (32) was obtained. Fluid depth variation with time was demonstrated. It was also noted that the applied field and electrical conductivity are inversely proportional to the velocity field and that as the electromagnetic forces increase the fluid in the tank drains slow and clearly complete drainage takes more time. The presented results are validated by assuming $\sigma B_{0}{ }^{2}=0$ in (34). The results of this work are compatible with those presented in [27], where the Bernoulli's equation was used.

\section{REFERENCES}

[1] D. D. Joye, B. C. Barrett, "The tank drainage problem revisited: Do these equations actually work?", The Canadian Journal of Chemical Engineering, Vol. 81, No. 5, pp. 1052-1057, 2003

[2] A. N. Hrymak, "Applied fluid mechanics by T. C. Papanastasiou, Prentice-Hall, Englewood Cliffs, NJ, 1994, 520 pp.”, AIChE Journal, Vol. 41, No. 10, pp. 2342-2342, 1995

[3] R. B. Bird, W. E. Stewart, E. N. Lightfoot, Transport phenomena, John Wiley and Sons, 1961

[4] E. J. Crosby, Experiments in transport phenomena, Wiley, 1961 
[5] D. Hanesian, A. Perna, A laboratory manual for fundamentals of engineering design, chemical engineering module: Measurements laboratory, New Jersey Institute of Technology, 1995

[6] N. D. Nevers, Fluid mechanics for chemical engineers, McGraw-Hill Education, 2005

[7] J. O. Wilkes, Fluid mechanics for chemical engineers with microfluidics and CFD, Prentice Hall, 2006

[8] S. Channer, K. N. Memon, A. A. Ghoto, A. M. Siddiqui, S. F. Shah, "Analytical solutionof lift for thin film flow for phan thien tanner fluid", Sindh University Research Journal, Vol. 51, No. 2, pp. 215-222, 2019

[9] L. K. Forbes, G. C. Hocking, "Unsteady draining flows from a rectangular tank", Physics of Fluids, Vol. 19, No. 8, Article ID 082104, 2007

[10] L. K. Forbes, G. C. Hocking, "Unsteady draining of a fluid from a circular tank", Applied Mathematical Modelling, Vol. 34, No. 12, pp. 3958-3975, 2010

[11] G. V. S. K. Reddy, C. V. Subbarao, "Comparison of efflux times between cylindrical and spherical tank through an exit pipe", International Journal of Engineering and Applied Sciences, Vol. 3, No. 2, pp. 61-68, 2011

[12] C. V. Subbarao, P. S. Rao, G. M. J. Raju, V. S. R. K. Prasad, "Slow draining of large spherical tank under gravity", Elixir International Journal, Vol. 50, pp. 10346-10348, 2012

[13] C. V. Subbarao, Y. P. Yadav, P. King, "Drag reduction by surfactant solutions in gravity driven flow systems", Iranian Journal of Chemistry and Chemical Engineering, Vol. 32, No. 2, pp. 119-123, 2013

[14] C. Y. Wang, "On a class of exact solutions of the navier-stokes equations", Journal of Applied Mechanics, Vol. 33, No. 3, pp. 696-698, 1966

[15] A. A. Mahessar, A. L. Qureshi, A. N. Laghari, S. Qureshi, S. F. Shah, F. A. Shaikh, "Impact of Hairdin, Miro Khan and Shahdad Kot drainage on Hamal dhand, Sindh", Engineering, Technology \& Applied Science Research, Vol. 8, No. 6, pp. 3652-3656, 2018

[16] K. N. Memon, A. M. Siddiqui, S. F. Shah, "Exact solution of tank drainage for Newtonian fluid with slip condition", Sindh University Research Journal, Vol. 49, No. 2, pp. 283-288, 2017

[17] S. Islam, K. N. Memon, A. M. Siddiqui, S. F. Shah, "Analytical solution of tank drainage for electrically conducting power law fluid", available at: https://www.preprints.org/manuscript/201802.0033/v1, 2018

[18] K. Afanasiev, A. Munch, B. Wagner, "Landau-Levich problem for nonNewtonian liquids", Physical Review E, Vol. 76, No. 3, Article ID 036307, 2007

[19] N. Ali, A. Abbasi, I. Ahmad, "Channel flow of Ellis fluid due to peristalsis", AIP Advances, Vol. 5, No. 9, Article ID 097214, 2015

[20] K. N. Memon, S. F. Shah, A. M. Siddiqui, "Exact solution of unsteady tank drainage for Ellis Fluid", Journal of Applied Fluid Mechanics, Vol. 11, No. 6, pp. 1629-1636, 2018

[21] M. R. Mohyuddin, T. Gotz, "Resonance behaviour of viscoelastic fluid in Poiseuille flow in the presence of a transversal magnetic field", International Journal for Numerical Methods in Fluids, Vol. 49, No. 8, pp. 837-847, 2005

[22] J. J. V. Rossum, "Viscous lifting and drainage of liquids", Applied Scientific Research, Section A, Vol. 7, pp. 121-144, 1958

[23] I. Pop, M. Kumari, G. Nath, "Conjugate MHD flow past a flat plate", Acta Mechanica, Vol. 106, pp. 215-220, 1994

[24] S. Abel, P. H. Veena, K. Rajgopal, V. K. Pravin, "Non-Newtonian magnetohydrodynamic flow over a stretching surface with heat and mass transfer", International Journal of Non-Linear Mechanics, Vol. 39, No. 7, pp. 1067-1078, 2004

[25] M. Abdullah, N. Saada, "Free convection MHD couette flow with application of periodic temperature and constant heat flux on walls", Engineering, Technology \& Applied Science Research, Vol. 9, No. 2, pp. 4007-4011, 2019

[26] G. F. Round, V. K. Garg, Applications of fluid dynamics, E. Arnold, 1986 